\title{
LQG/LTR CONTROL OF THE DISTRIBUTED COLLECTOR FIELD OF A SOLAR POWER PLANT
}

\author{
F.R. Rubio, F. Gordillo and M. Berenguel ${ }^{1}$
}

\author{
Dpto. Ingeniería de Sistemas y Automática, Escuela Superior de Ingenieros, \\ Universidad de Sevilla. Avda. Reina Mercedes s/n, 41012-Sevilla (Spain) \\ Telf. 34-5-4556876. Fax: 34-5-4556849/4629205. E-mail: rubio@esi.us.es
}

\begin{abstract}
This paper describes a LQG/LTR controller for a solar distributed collector field. When designing a LQG controller it is easy to take into account the trade-off between quick response and avoiding oscillations which is typical of many control problems. The improved robustness reached with the loop transfer recovery LTR allows for good behaviour of the resulting system even when the working conditions are far from the ones the LQG controller has been designed for. The controller also employs serial compensation to cope with measurable external disturbances. The real resulting behaviour is shown by means of experimentation on the plant.
\end{abstract}

Keywords. Solar power plant, Optimal control, Loop transfer recovery, Robust control.

\section{INTRODUCTION}

Solar power provides an energy source which varies independently and cannot be adjusted to suit the desired demand. Although solar radiation does have predictable seasonal and daily cyclic variations it is also affected by unpredictable variations caused by atmospheric conditions such as cloud cover, humidity and air transparency. When using solar power as a primary heat source the major aim is to maximize the usage of the available energy maintaining desired operating conditions for the process involved. With the distributed collector field concerned, solar radiation is focussed by mirrors onto a pipe through which oil flows. The energy collected is transferred to a storage tank, which can be tapped when conditions demand, onto either a steam generator for electrical power generation or the heat exchanger of a desalination plant.

1 This work has been founded by CICYT under grant TAP-95-0307
The prime control requirement is to maintain the outlet oil temperature of the field at a constant value. Since solar radiation cannot be adjusted this can only be achieved by adjusting the oil flow, and the daily solar power cycle characteristic is such that the oil flow has to change substantially during operation. This leads to significant variations in the dynamic characteristics of the field such as response rate and time delay. Some control schema have been tested at the plant during the last five years (Camacho et al., 1994a), (Camacho et al., 1994b). The LQG/LTR control approach allows changes in dynamic characteristics to be compensated for whilst maintaining the desired control performance with a high degree of robustness.

This article presents a control structure in which the controller is an LQG. This type of controller works well when the state is accessible but its robustness is weakened when an observer is introduced. In order to solve this problem it is proposed that the design should be modified so as to recuperate the stability margins in ef- 
fect when the state is accessible. In the literature this procedure is called Loop Transfer Recovery (LTR). The use of this methodology leads to more robust controllers (Doyle and Stein, 1979), (Rubio and Aracil, 1990). The application of this approach is covered and results obtained by simulation using a non-linear model of the plant and results from the plant itself are presented.

The paper is organized as follows: in section 2 a brief description of the distributed collector field is given. Section 3 is dedicated to presenting the control structure used. In section 4 the controller design procedure and simulation studies are given. Section 5 presents results obtained when applying the LQG/LTR controller to the distributed solar collector field. Conclusions are given in section 6 .

\section{SYSTEM DESCRIPTION}

The system considered corresponds to the ACUREX distributed collector field of the SSPS solar plant, located in Almería (Southern Spain). The main objective of the distributed field is to collect solar energy by heating oil passing through the field. The field consists of 480 distributed solar collectors. These collectors are arranged in 20 rows which form 10 parallel loops, as indicated schematically in Fig. 1, and lie along an east west axis.

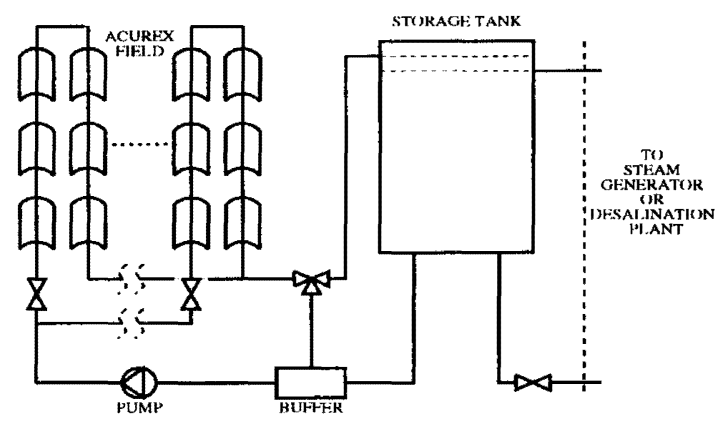

Fig. 1. Schematic Diagram of Collector Field

The collector uses a parabolic surface to concentrate a direct normal beam onto the receiver tube which is located at the focal point of the parabola. The heat transfer fluid is pumped through the receiver tube and picks up the heat transferred though the receiver tube walls.

The field is also provided with a tracking system which causes the mirrors to revolve around an axis parallel to that of the pipe enabling the varying inclination of the sun to be followed. The cold inlet oil is extracted from the bottom of the storage tank and is passed through the field using a pump located at the field inlet. This fluid is heated and then introduced into a storage tank to be used for electrical energy generation. The system is provided with a three way valve located at the field outlet that allows the oil to be recycled whithin the field until its outlet temperature is adequate for entering into the top of the storage tank. Each of the loops mentioned above is formed by four twelve module collectors, suitably connected in series. The loop is 172 metres long, the active part of the loop measuring 142 metres and the passive part 30 metres.

\section{CONTROL STRUCTURE}

High order linear models of the field have been obtained taking into account the frequency response of the plant, showing antiresonance characteristics (Camacho et al., $1994 b$ ), but in the development of the robust LQG/LTR controller a low order model is used to show that even when the model uncertainty is high, the robust control scheme proposed provides very good results and a low order controller.

In this way, a simple linear model for control purposes relating changes in fluid flow, the adjustable input variable, to changes in outlet temperature is used (Camacho et al., 1992). Observations of step responses obtained from the plant indicate that in the continuous time domain behaviour can be closely approximated by a first order transfer function with a time delay:

$$
g(s)=e^{-s \tau_{d}} \frac{K}{(1+\tau s)}
$$

The time delay $\tau_{d}$, time constant $\tau$ and gain $K$ of the system vary with the oil flow-rate and at the lower operational flowrate the delay is approximately twice that of at the maximum flow rate. It has been shown (Camacho et al., 1992), that one way of accommodating this variation in time delay is to use a model of the form:

$$
g(z)=z^{-2} \frac{\left(b_{0}+b_{1} z^{-1}\right)}{\left(1-a z^{-1}\right)}
$$

\subsection{Series Compensation}

In order to define input and output signals of the LQG/LTR, some aspects have to be taken into account.

The control signal used is the oil flow. The outlet temperature of the plant, however, is also influenced by changes in system variables such as solar radiation and fluid inlet temperature. Hence, dynamically, the outlet temperature $T_{o}$ can be expressed as a nonlinear function $f$ of oil flow $u_{f}$, solar radiation $I$ and inlet temperature $T_{i n}: T_{o}=f\left(u_{f}, I, T_{i n}\right)$. The mathematical model which accounts for these additional influences dynamically, even when linearized, is complex, especially when 
considering that the variation of the inlet temperature $\left(T_{i n}\right)$ produces a change in the outlet temperature after a variable delay time (depending on the flow).

One simple approach which reduces the complexity of the model is to make use of the known operating characteristics of the field. Studies have been carried out which incorporated approximate transfer functions in the feedforward term but the benefits provided were not considered to justify the added complexity or uncertainty involved. So, in order to account for the disturbances, a series feedforward controller was introduced (Camacho et al., 1992), directly calculated from steady-state relationships (steady-state energy balance), which makes an adjustment in the flow input, aimed at eliminating the change in outlet temperature caused by the variations in solar radiation and inlet temperature. The calculation employed is:

$$
u_{f}=\frac{0.7869 I-0.485(u-151.5)-80.7}{\left(u-T_{i n}\right)}
$$

where $u_{f}$ is the oil flow, $u$ is the temperature set-point given by the LQG/LTR, $T_{i n}$ is the inlet oil temperature and $I$ is the effective solar radiation. The feedforward is placed in series with the LQG/LTR. and thus, the output signal of the LQG/LTR controller is the increment in the set point temperature for the feedforward term and not the oil flow (this is calculated by the feedforward controller).

\section{$3.2 L Q G / L T R$ Controller}

It has been observed that the Linear Quadratic Gaussian Controller (LQG) method worked well when very precise mathematical models were used, but the method was extremely sensitive to imprecisions in the parameters and to structural modifications. One outstanding property is that the closed loop transfer function obtained when the state is directly accessible, so that the Kalman filter (KBF) is not necessary, is the same as if this last module were included. That is, the closed loop is the same as if the observer were inexistent; there is no influence on the global transfer function of the system. This gave the idea that the observer was irrelevant in the design of the closed loop system. However, as was discovered at the end of the seventies, although it is true that the observer does not affect the closed loop transfer function it noticeably affects the open loop transfer function. This influence can be extremely damaging to the robustness of the system (in fact it can greatly reduce the stability margins).

\section{The LQR and LQG regulators}

Given the plant model:

$$
\begin{aligned}
\mathbf{x}(k+1) & =\mathbf{A x}(k)+\mathbf{B u}(k) \\
\mathbf{y}(k) & =\mathbf{C x}(k)
\end{aligned}
$$

The purpose of the LQR regulator is to determine the control signal $u$ in order to minimize the following functional:

$$
\begin{gathered}
\mathcal{J}=\mathbf{x}_{N}^{T} \mathbf{H}_{c} \mathbf{x}_{N}+\sum_{k=0}^{N-1}\left(\mathbf{x}^{T}(k) \mathbf{Q}_{c} \mathbf{x}(k)+\mathbf{u}^{T}(k) \mathbf{R}_{c} \mathbf{u}(k)\right) \\
\text { with: } \quad \mathbf{Q}_{c}=\mathbf{Q}_{c}^{T} \geq 0, \quad \mathbf{R}_{c}=\mathbf{R}_{c}^{T} \geq 0
\end{gathered}
$$

$\mathbf{x}$ and $\mathbf{x}_{N}$ being the state and the final state vectors respectively. The LQG problem is presented which is based on the separation theorem, according to which the control strategy can be divided into two parts:

(1) An optimum control problem, from which the regulation by feedback of state variables is obtained.

$$
\mathbf{u}=-\mathbf{K}_{c} \hat{\mathbf{x}}
$$

$\mathbf{K}_{c}$ is found by iteration of the well known Riccati equation.

(2) An optimum filtering problem which solves the observation using the Kalman filter, whose corresponding dynamic is given by the system:

$$
\begin{aligned}
\hat{\mathbf{x}}(k+1)= & \mathbf{A} \hat{\mathbf{x}}(k)+\mathbf{B u}(k)+\mathbf{K}_{o}(k+1) \quad[\mathbf{y}(k+1)- \\
& \mathbf{C}(\mathbf{A} \hat{\mathbf{x}}(k)+\mathbf{B} \mathbf{u}(k))]
\end{aligned}
$$

where $\mathbf{K}_{o}$ is obtained from a Riccati equation dual to the previous one.

The resulting structure is shown in Fig. 2.

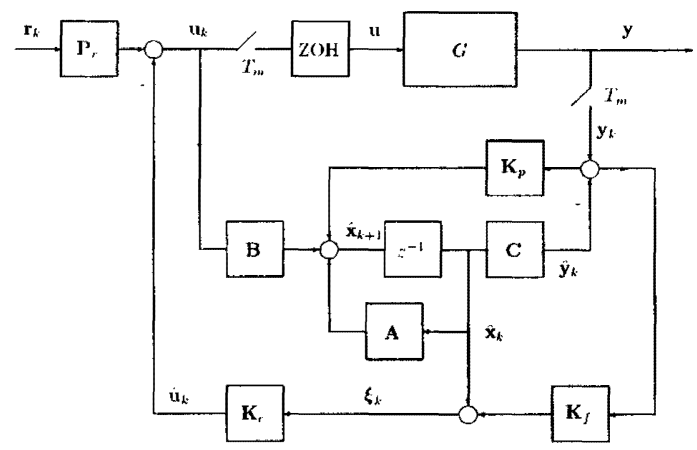

Fig. 2. Block diagram of the LQG regulator

\section{Loop Transfer Recovery (LTR)}

Asymptotic recovery techniques have been developed for continuous time, minimum phase systems (Doyle and Stein, 1979). There are design techniques which allow for excellent robustness properties. To dispose of a similar procedure for discrete time systems is, therefore, desirable, even though the LQR discrete controller does not 
have the same properties as present in the continuous case, such as stability margins.

The solution of the controller LQR is a linear feedback of the state vector and gives rise to the open loop transfer function: $G_{L Q R}(z)=\mathbf{K}_{c} \Phi \mathbf{B}$ where $\Phi=(z \mathbf{I}-\mathbf{A})^{-1}$.

By mathematical manipulation in Fig. 2, the transfer function of the open loop $G_{L Q G}(z)$, can be obtained and this depends on the parameters of the control law and of the Kalman filter, which in turn depend on the costs assigned to resolving the control and observation problems. It is a well known fact that the closed loop transfer function never varies, thus the closed loop temporary specifications solved by the application of the control law will continue to be fulfilled. However, the open loop transfer function becomes:

$$
\begin{array}{r}
G_{L Q G}(z)=\left[\mathbf{K}_{c}\left(\mathbf{I}-\mathbf{K}_{o} \mathbf{C}\right)\left[z \mathbf{I}-\left(\mathbf{A}-\mathbf{B K}_{c}\right)\left(\mathbf{I}-\mathbf{K}_{o} \mathbf{C}\right)\right]^{-1}\right. \\
\left.\left(\mathbf{A}-\mathbf{B} \mathbf{K}_{c}\right) \mathbf{K}_{o}+\mathbf{K}_{c} \mathbf{K}_{o}\right] \mathbf{C \Phi B}
\end{array}
$$

With this situation the stability margins have changed, and in most cases this variation usually goes into action by greatly deteriorating the phase margin of the compensated system, causing it the consequent loss of robustness.

To solve the problems presented by the deterioration of robustness caused by the introduction of the observer, the method known as LQG/LTR has been proposed. The idea of loop transfer recovery at the input LTR-i is to modify the covariance matrices of the Kalman Filter so that the open loop transfer function of the system with the LQG controller approximates $G_{L Q R}(z)$, which has good robustness properties. This can be achieved, in the case of minimum-phase systems in the following way:

$$
\begin{aligned}
& \mathbf{Q}_{o}=\mathbf{Q}_{o}^{0}+q^{2} \mathbf{B B}^{T} \\
& \mathbf{R}_{o}=\mathbf{R}_{o}^{0}
\end{aligned}
$$

As $q^{2} \rightarrow \infty$, the open loop transfer function of the LQG problem approaches the LQR one.

In the case of discrete systems (Maciejowski, 1985), it can be shown that for minimum phase systems such as $\operatorname{det}(\mathbf{C B}) \neq 0$ and using the filtered version of the Kalman filter as observer, perfect recuperation is obtained acting on the parameters of the LQR. In any case, when these conditions are not fulfilled, the same procedure usually leads to improvements of robustness.

\section{CONTROLLER DESIGN}

The effect of the loop transfer recovery on the increase in robustness of the controlled system has been anal- ysed first. The controlled system could be expected to be less sensitive to changing dynamics due to set point variations or unmodelled dynamics in this way.

In order to carry out the design of the LQG/LTR controller a linear model must be considered first. In this case, the reduced order model proposed in (Camacho et $a l ., 1992)$ and considered in section 3 has been employed.

In order to show the advantages of the LTR method, the controller has been designed considering a model of the plant: $G(z)=0.1 z^{2} /\left(1-0.9 z^{-1}\right)$, which corresponds to high flow operating conditions. The model has been augmented with an integrator in order to avoid static errors and the controllable canonical form has been used. The weighting matrices of the $\mathrm{LQ}$ problem have been used equal to: $\mathbf{Q}_{c}=\mathbf{C}^{T} \mathbf{C} ; \mathbf{R}_{c}=17$, where $\mathbf{C}$ is, as usual, the output matrix of the internal description of the plant. The value $\mathbf{R}_{c}=17$ has been chosen from simulation studies in order to achieve good tracking characteristics.

Since it is not possible to obtain an estimation of the noises corresponding to the Kalman filter (amongst other facts, the internal description used has no physical meaning), the covariance matrices of the Kalman filter have been chosen arbitrarily. In concrete, a LTR-i form has been used: $\mathbf{Q}_{o}=\mathbf{B B}^{T} ; \mathbf{R}_{o}=\rho$ where $\mathbf{B}$ is, as usual, the input matrix of the internal description of the plant and $\rho$ is a design parameter. According to the LQG/LTR theory, decreases of $\rho$ will, probably, result in robustness increases.

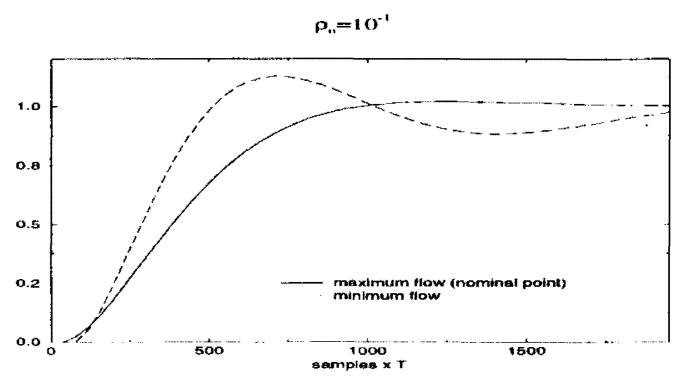

Fig. 3. Simulation with $\rho=0.1$

The behaviour of the simplified linear model $G(z)$ with a design value of $\rho=0.1$ has been tested both at the design point (maximum flow) and at the extreme point (minimum flow, which corresponds to the same transfer function augmented with $z^{-1}$ (Camacho et al., 1992)). Simplifying assumptions have been made, because fixed values of $b_{0}, b_{1}$ and $a$ have been considered in the reduced order model shown in section 3. As can be seen in Fig. 3 , the behaviour of the controlled system is influenced by changes at operating point. If a value of $\rho=10^{-10}$ is chosen in the design, the controlled system is less sensi- 
tive to changes at the operating point (Fig. 4), as is to be expected.

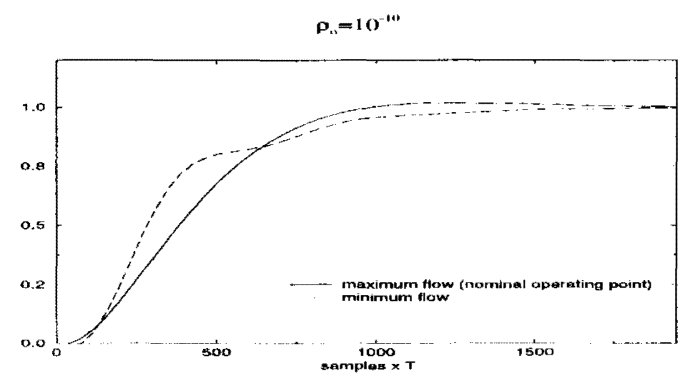

Fig. 4. Simulation with $\rho=10^{-10}$

\subsection{Simulation studies}

In order to develop a LQG/LTR controller for the distributed solar collector field, a model corresponding to an identification of the step-response of the system at a concrete operating condition (oil flow equal to $8 \mathrm{l} / \mathrm{s}$ ) has been chosen. This model would be very different under other conditions, but, due to the robustness of the controller, stability and good set point tracking is obtained under different operating conditions. The model results to be equal to:

$$
G_{1}(z)=\frac{0.027 z+0.052}{z^{3}-0.912 z^{2}}
$$

As mentioned previously, the model is augmented with an integrator and the controllable canonical form is used as internal description of $G(z)=\frac{z}{z-1} G_{1}(z)$. The weighting matrices of the $L Q$ problem stated above have been used.

In Fig. 5 the Nyquist diagram of the model used for design purposes with the LQG controller is represented, with different values of $\rho$. It can be seen that, as $\rho$ decreases, the phase margin of the controlled system increases (or any other robustness measure).

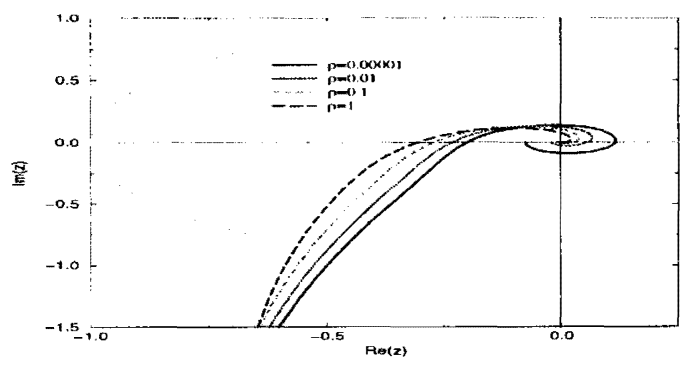

Fig. 5. Nyquist diagram of the model with the LQG/LTR controller for different values of $\rho$
The plant can be described by a set of nonlinear distributed parameter equations describing energy and mass balance. A nonlinear distributed parameter model was developed so that the control scheme could be tested by simulation. Under certain conditions (Berenguel et al., 1994), the temperature in the field can be given by the following equations:

$$
\begin{gathered}
\rho_{m} C_{m} A_{m} \frac{\partial T_{m}}{\partial t}=I n_{o} D-H_{1} G\left(T_{m}-T_{a}\right)-L H_{t}\left(T_{m}-T_{f}\right) \\
\rho_{f} C_{f} A_{f} \frac{\partial T_{f}}{\partial t}+\rho_{f} C_{f} \dot{q} \frac{\partial T_{f}}{\partial x}=L H_{t}\left(T_{m}-T_{f}\right)
\end{gathered}
$$

where the subindex $m$ refers to the metal and $f$ to the fluid. The rest of the parameters are as follows: $\rho$ : density, $C$ : field capacity, $A$ : transversal area, $T$ : outlet temperature, $I$ : solar radiation, $n_{o}$ : optical efficiency, $H_{1}$ : overall thermal loss coefficient, $D$ : mirror width, $H_{t}$ : coefficient of metal fluid transmission, $G$ : exterior diameter of the pipe line, $L$ : inner diameter of the pipe line, $\dot{q}$ : oil flow rate. These equations are only applicable to the active zones of the field, that is, those parts of the pipe line where solar radiation is collected. Parts of the field, passive zones, exist where it is not possible to collect solar energy due to geometrical conditions, as is the case of the joints between the modules. These zones constitute a considerable part of the field and they are characterized by having nil irradiance and different loss constants.

The above equations were used to simulate the system in a computer dividing one of the loops into one hundred pieces and using a model of concentrated parameters for each piece. The model was contrasted to the real data obtained from the field (Berenguel et al., 1994). The parameters of the model were adjusted so that they reproduced the behaviour of the system.

Fig. 6 corresponds to a simulation (set point and outlet oil temperatures) of the same controllers with the distributed nonlinear model of the plant.

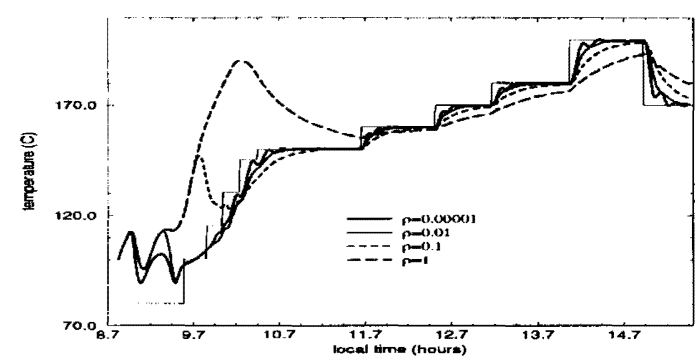

Fig. 6. Simulation of the LQG/LTR controller for different values of $\rho$

The value of $\rho=0.00001$ has been chosen to obtain a good trade-off between robustness and desired shape 
of the step response. Results of simulations are good compared to other controller approaches including adaptive schemes (Camacho et al., 1992), (Camacho et al., $1994 a$ ). In this case, the lack of adaptive behaviour is compensated for by the high robustness of the controller.

\section{PLANT RESULTS}

The proposed control scheme has been tested at the solar plant. Above all, one aspect in the development of the LQG/LTR controller used should be stressed, that is the excellent results obtained in the first tests performed at the plant. This fact can be observed in the figure showing the first test performed at the plant. Fig. 7 (outlet oil temperature, inlet oil temperature, set point temperature, solar radiation and oil flow respectively) corresponds to a step-response test (19/09/95), covering a wide range of oil-flow conditions (from 1.8 to 6 $\mathrm{l} / \mathrm{s}$ ). As can be seen, good set-point tracking and disturbance rejection was obtained, with null steady state error (due to the fact that the controller is implemented including integral action). The rise time was of about 8 minutes without overshoot in a change of 10 degrees at the set point.

\section{CONCLUSIONS}

A method to design a controller with a LQG/LTR structure has been shown. This method has proved to have better properties of robustness than the original LQG method making it possible to use the $\mathrm{LQG}$ in more situations. The validity of this controller has been proved by simulation and by different tests on a solar power plant.

\section{ACKNOWLEDGMENT}

The authors would like to thank CIEMAT and the personnel of the Solar Platform of Almería, especially D. Pedro Balsa, for their collaboration in carrying out the tests.

\section{REFERENCES}

Berenguel, M., E. F. Camacho and F.R. Rubio (1994). Simulation Software Package for the Acurex Field. Dpto. de Ingeniería de Sistemas y Automática, ESI Sevilla (Spain), Internal Report.

Camacho, E.F., F.R. Rubio and F.M. Hughes (1992). Self-tuning control of a solar power plant with a distributed collector field. IEEE Control Systems Magazine pp. 72-78.
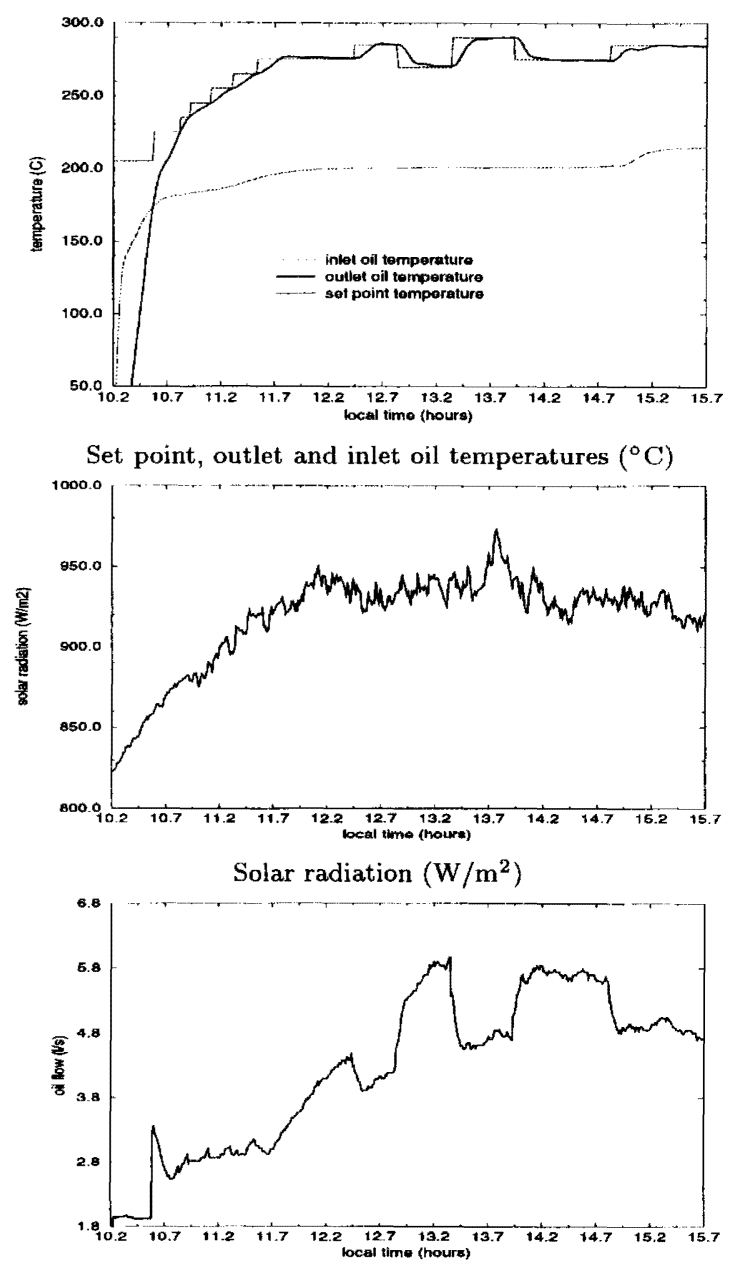

Oil flow $(1 / \mathrm{s})$

Fig. 7. Test carried out with the LQG/LTR on 19th September, 1995

Camacho, E.F., M. Berenguel and C. Bordóns (1994a). Adaptive generalized predictive control of a distributed collector field. IEEE Trans. on Control Systems Technology 2(4), 462-467.

Camacho, E.F., M. Berenguel and F.R. Rubio (1994b). Application of a gain scheduling generalized predictive controller to a solar power plant. Control Engineering Practice 2(2), 227-238.

Doyle, J.C. and G. Stein (1979). Robustness with observers. IEEE Trans. on Automatic Control AC24(4), 607-611.

Maciejowski, J.M. (1985). Asymptotic recovery for discrete-time systems. IEEE Trans. on Automatic Control AC-30(6), 602-605.

Rubio, F.R. and J. Aracil (1990). Classical/modern design of a tracking control system. In: Proc. of the IEEE International Conference on Systems Engineering. 